\title{
Timing of Toenail Collection and Concentrations of Metals in Pancreatic Cancer. Evidence Against Disease Progression Bias
}

\author{
José Pumarega ${ }^{1,2,3} \cdot$ Judit Camargo ${ }^{1,3} \cdot$ Magda Gasull1,2,3,4 $^{1}$ Andrew F. Olshan ${ }^{5} \cdot$ Amr Soliman $^{6} \cdot$ Yu Chen ${ }^{7}$. \\ David Richardson $^{5}$. Juan Alguacil ${ }^{2,8}$. Charles Poole ${ }^{5}$. Leonardo Trasande ${ }^{7,9,10} \cdot$ Miquel Porta $^{1,2,3,5,9}$ (1) for the \\ PANKRAS II Study Group
}

Received: 1 March 2021 / Revised: 27 September 2021 / Accepted: 30 September 2021 / Published online: 22 October 2021

(C) The Author(s), under exclusive licence to Springer Nature B.V. 2021

\begin{abstract}
Trace elements such as cadmium, arsenic, zinc or selenium increase or decrease risk of a wide range of human diseases. Their levels in toenails may provide a measure of mid-term intake of trace elements for studies in humans. However, in biologically and clinically aggressive diseases as pancreatic cancer, the progression of the disease could modify such concentrations and produce reverse causation bias. The aim was to analyze the influence of specific time intervals between several clinical events and the collection of toenails upon concentrations of trace elements in patients with pancreatic cancer. Subjects were 118 incident cases of pancreatic adenocarcinoma prospectively recruited in eastern Spain. Toenails were collected at cancer diagnosis, and soon thereafter interviews were conducted. Information on cancer signs and symptoms was obtained from medical records and patient interviews. Levels of 12 trace elements were determined in toenail samples by inductively coupled plasma mass spectrometry. General linear models adjusting for potential confounders were applied to analyze relations between $\log$ concentrations of trace elements and the time intervals, including the interval from first symptom of cancer to toenail collection (iST). Toenail concentrations of the 12 trace elements were weakly or not influenced by the progression of the disease or the diagnostic procedures. Concentrations of aluminum were slightly higher in subjects with a longer iST (age, sex and stage adjusted geometric means: $11.44 \mathrm{vs.} 7.75 \mu \mathrm{g} / \mathrm{g}$ for iST $>120$ days vs. $\leq 40$ days). There was a weak inverse relation of iST with concentrations of zinc and selenium (maximum differences of about $20 \mathrm{and} 0.08 \mu \mathrm{g} / \mathrm{g}$, respectively). Conclusions: concentrations of the trace elements were weakly or not influenced by the development of the disease before toenail collection. Only concentrations of aluminum increased slightly with increasing iST, whereas levels of zinc and selenium decreased weakly. Even in an aggressive disease as pancreatic cancer, toenail concentrations of trace elements may provide a valid measure of mid-term intake of trace elements, unaffected by clinical events and disease progression.
\end{abstract}

Keywords Pancreatic neoplasms $\cdot$ Biomarkers $\cdot$ Symptoms $\cdot$ Toenails

$\begin{array}{ll}\text { Abbreviations } \\ \text { BMI } & \text { Body mass index } \\ \text { CI } & \text { Confidence interval } \\ \text { CT } & \text { Computerized axial tomography }\end{array}$

Judit Camargo and Magda Gasull have contributed equally to this work.

Members of the Multicentre Prospective Study on the Role of KRAS and other Genetic Alterations in the Diagnosis, Prognosis and Etiology of Pancreatic Cancer (PANKRAS II) Study Group are mentioned in previous publications.

Miquel Porta

mporta@imim.es

Extended author information available on the last page of the article
ERCP Endoscopic retrograde cholangiopancreatography GM Geometric mean

iSB Interval from first symptom of pancreatic cancer to blood extraction

iSD Interval from first symptom of pancreatic cancer to diagnosis of pancreatic cancer

iST Interval from first symptom of pancreatic cancer to toenail collection

OCs Organochlorine compounds

PDAC Pancreatic ductal adenocarcinoma

SD Standard deviation

SRM Standard reference material

TNM Tumor-node-metastasis system 


\section{Introduction}

Trace elements (in particular chromate, nickel, cadmium and arsenic compounds) are well-known carcinogens, and dietary contaminants (Chervona et al. 2012). They act by diverse mechanisms, including oxidative stress, they inhibit zinc-finger DNA repair machinery, and induce polyadenylation of canonical histones (Huang et al. 2004; Brocato et al. 2015; Huff et al. 2007). While these metals are best known for induction of lung, liver, colon and prostate cancer, their contribution to other cancers is under scrutiny.

Increases in pancreatic cancer incidence have raised substantial concern about environmental contributions, and suggest opportunities for prevention (Fernandez et al. 1994; Ojajärvi et al. 2000; Porta et al. 2003a). While multiple studies have examined occupational exposures as risks, particularly for cadmium (Chen et al. 2015), few have examined exposures in general populations, which are subject to chronic, low-level exposure due to ubiquitous contamination.

Previous studies suggest an association between pancreatic cancer risk and concentrations of trace metals such as cadmium, lead, arsenic, and selenium measured in toenails (Gómez-Tomás et al. 2019; Camargo et al. 2019; Amaral et al. 2012). However, the influence of clinical events (signs and symptoms, time until diagnosis, diagnostic tests, tumor stage) on body concentrations of trace elements has not been evaluated in pancreatic cancer, other neoplasms, or other diseases.

Information on concentrations of trace elements in nails has long been used in humans to cost-effectively measure exposure and internal dose. In healthy and physically stable individuals, and compared to blood, urine, or hair samples, levels in toenails of mercury, manganese, and arsenic appear to reflect better the corresponding concentrations in the organism for a time frame from a few months to a year preceding sample collection (Amaral et al. 2012; He 2011; Gutiérrez-González et al. 2019; Salcedo-Bellido et al. 2021; Signes-Pastor et al. 2020; Longnecker et al. 1993; Garland et al. 1993; Hunter et al. 1990; Hopps 1977; Sukumar 2006; Ntihabose et al. 2018; Marchiset-Ferlay et al. 2012).

Little is known about the toxicokinetic and toxicodynamic profiles of trace elements during tumor formation and progression, particularly about the methodological implications for research on their possible etiologic roles in cancer (Koedrith et al. 2013; Chen and Costa 2017).

Studies that employ biomarkers of exposure in individuals who already have (asymptomatic or symptomatic) cancer may be biased by metabolic processes inherent to the disease. This has been demonstrated for organochlorine compounds (OCs) in blood samples of individuals diagnosed with pancreatic cancer, in whom the time interval between the first symptom of the disease and blood extraction (iSB) was associated with serum concentrations of total lipids and OCs (Porta et al. 2007, 2008, 2009). Concentrations of the highly lipophilic OCs were also associated with signs and symptoms of the disease and with tumor's stage at diagnosis. Hence, during the progression of pancreatic cancer and other diseases, patients experience weight loss, cholestasis, and other clinical and pathophysiological changes that alter concentrations of lipids, lipophilic nutrients, and lipophilic environmental compounds. Disease progression bias is thus a form of reverse causation. It results in a lack of etiologic significance of the disease-altered exposure biomarkers (Porta et al. 2007; Porta et al. 2008; Porta et al. 2009; Porta et al. 2014; Porta et al. 2005; Porta et al. 2021; Porta 2001; Gasull et al. 2019). This conceptual and empirical framework provides the rationale to hypothesize that pancreatic cancer progression might also modify concentrations of trace elements in toenails (Gómez-Tomás et al. 2019; Camargo et al. 2019; Amaral et al. 2012; Crous-Bou 2009).

Therefore, the aim of the present study was to analyze the influence of specific time intervals between several clinical events and the collection of toenails upon concentrations of trace elements in patients with pancreatic cancer.

\section{Materials and Methods}

\section{Study Population}

Methods of the PANKRAS II study have been previously described (Gómez-Tomás et al. 2019; Camargo et al. 2019; Amaral et al. 2012; Porta et al. 2007, 2008, 2009, 2005, 2020, 1999, 2000; Crous-Bou 2009; Soler et al. 1999). Briefly, subject recruitment took place between 1992 and 1995 at five general hospitals in the Mediterranean part of Spain, where 185 incident cases of PDAC were prospectively identified. The present report is based on 118 incident cases of PDAC with toenail samples obtained and metal concentrations analyzed (Amaral et al. 2012). There were no significant differences between the 118 patients with and the 67 patients without available trace elements concentrations for a broad range of sociodemographic and clinical variables, including age, sex, social class, education, occupation, smoking, coffee consumption, duration of interview, interval from first symptom of pancreatic cancer to diagnosis, and interval from first symptom to blood extraction (GómezTomás et al. 2019; Camargo et al. 2019; Porta et al. 2020) (Supplementary Table 1). The Ethics Committees of participating hospitals approved the study protocol, and patients gave informed consent to be included in the study. 


\section{Personal Interviews and Information on Symptoms}

A structured form was used to collect clinicopathological information from medical records, including details on semiology, diagnostic procedures, laboratory results and followup (Soler et al. 1999; Porta et al. 2000). Follow-up extended for 17.5 years (Porta et al. 2020). Hospital discharge diagnoses and the tumor clinical stage were also recorded. The tumor's clinical stage at diagnosis was classified according to the tumor-node-metastasis (TNM) system. Diagnostic tests included ultrasound scan, computerized axial tomography (CT), fibrogastroscopy, endoscopic retrograde cholangiopancreatography (ERCP), gammagraphy, laparoscopy, and exploratory laparotomy (Porta et al. 2007). When a diagnostic procedure was performed more than once, the physician who abstracted the data chose the more informative result. Over $88 \%$ of the PDAC patients were interviewed face-to-face by trained monitors during their hospital stay, close to the time of diagnosis (Camargo et al. 2019; Amaral et al. 2012; Porta et al. 2020).

Detailed information on the first symptom of cancer, including the date, and signs and symptoms of the disease was obtained from two sources: medical records (where they were registered by the attending physician at hospital admission), and interviews with patients (Porta et al. 2005; CrousBou 2009; Soler et al. 1999). The information was reviewed by two experienced oncologists and checked for consistency. If data elicited from the interview contradicted data abstracted from the medical record, the latter was taken as the consensus data (Porta et al. 2005). Pathology of all cases was independently reviewed by the study reference pathologists, who were unaware of the original diagnosis. A panel of clinical and surgical experts in gastrointestinal diseases reviewed hospital discharge diagnoses of all patients and, based on all clinical and pathological information available, including follow up, reached a consensual clinicopathological diagnosis (Porta et al. 2000).

\section{Analyses of Trace Element Concentrations}

Nail clippings from the larger toe were collected once per patient during the hospital stay when the cancer was diagnosed, and were stored at room temperature until the time of the analyses. Trace elements analyzed were cadmium, arsenic, selenium, nickel, lead, chromium, manganese, aluminum, iron, vanadium, copper, and zinc (Gómez-Tomás et al. 2019; Camargo et al. 2019; Amaral et al. 2012; Porta et al. 2020). After careful cleaning and washing to remove external contaminants, trace elements were quantified at the Trace Element Analysis Core (Dartmouth College, Hanover, New Hampshire, USA), using inductively coupled plasma mass spectrometry. Toenails were acid digested with Optima nitric acid (Fisher Scientific, St Louis, Missouri, USA) at $105^{\circ} \mathrm{C}$ followed by the addition of hydrogen peroxide and further heating of the dilution with deionized water. All sample preparation steps were recorded gravimetrically. As a quality control, each batch of analyses included six standard reference material (SRM) samples with known trace element content (GBW 07601, powdered human hair) and six analytical blanks, along with the study samples (Gómez-Tomás et al. 2019; Camargo et al. 2019; Amaral et al. 2012; Porta et al. 2020).

The within-assay coefficients of variation for SRM replicates were $<15 \%$ for aluminum, arsenic, copper, lead, manganese, selenium, and zinc; and $15-40 \%$ for cadmium, chromium, iron, nickel, and vanadium. The between-assay coefficients of variation for SRM replicates were $<15 \%$ for arsenic, lead, manganese, selenium, and zinc; $15-30 \%$ for aluminum, cadmium, copper, and nickel; and $>30 \%$ for chromium, iron, and vanadium (Supplementary Table 2). The amount of SRM used ranged from less than 10 to $50 \mathrm{mg}$ to mimic the mass of toenails. This small SRM sample mass may be the cause of some of the variability seen in the within- and between-batch SRM results.

Eight of the 12 trace elements were detected in all 118 subjects (i.e., the percentage of detection was 100\%), whereas the other four elements were detected in $99.2 \%$, $98.3 \%, 98.3 \%$, and $92.4 \%$ of subjects (Supplementary Table 2). Therefore, the corresponding percentage of participants below the detection limit was $0 \%$ for eight of the 12 trace elements, and $0.8 \%, 1.7 \%, 1.7 \%$, and $7.6 \%$ for the other four.

The lowest concentration of each element detected in a given subject is also shown in Supplementary Table 2. Given the very high percentages of detection, such concentration is the most meaningful information; it reflects the limit of detection of the instrumental technique for the individual metals.

\section{Statistical Analysis}

Univariate statistics were computed as customary to describe key patient and trace metal variables (Armitage et al. 2002; Kleinbaum et al. 1998). All time intervals were calculated using the date of the clinical or diagnostic event as reference. As an example, the interval between the first symptom of pancreatic cancer and the date of toenail collection is the time elapsed from the first symptom to toenail collection (iST). We analyzed the time intervals between toenail collection and: hospital admission, performance of diagnostic tests, blood extraction, diagnosis, treatment onset, and interview (Supplementary Tables 3 and 4).

Among patients with an iST $\leq 40$ days $48 \%$ of patients were in tumor stage I and $21 \%$ in tumor stage IV, while among patients with iST $>120$ days there were $16 \%$ of patients in tumor stage I and $37 \%$ of patients in stage IV. 
The median time of the interval from hospital admission to toenail collection was 15 days, and to diagnosis and blood extraction 0 days. The median iST was 70.5 days, with a range from one week to more than two years (11 to 763 days) (Fig. 1), and is similar to the median time interval between the first symptom of the disease and blood extraction (iSB), 72 days (167 patients); the distribution of these two intervals (iST and iSB) is virtually identical (Fig. 1). When only patients with both samples, blood and toenails, were considered $(N=117)$, the median iST and iSB were 71 and 70 days, respectively; in 84 such patients $(72 \%)$ the blood and toenails were collected on the same day.

Supplementary Fig. 1 shows the iST for each patient (all represented on the ' $y$ ' axis), with patients classified from shorter to longer interval. The color of the lines shows the number of diagnostic tests performed before the toenail collection. We collected about $60 \%$ of toenail samples during the first three months after symptom onset; at 6 months $80 \%$ of the samples had been collected. In $>75 \%$ of patients the toenail collection was performed during the first month following hospital admission (Supplementary Table 3). This timing is a remarkable logistic achievement for a study of a severe disease as pancreatic cancer, and it may be a benchmark for other studies. A total of 315 diagnostic tests were performed in the hospitals before the collection of toenails (median of 3 diagnostic tests per patient); over $60 \%$ of patients with iST $\leq 40$ days underwent three diagnostic tests before the collection (Supplementary Fig. 1).

Kruskal-Wallis' test and ANOVA's test were used to assess differences between metal concentrations by iST categorized as $\leq 40$ days, 41 to 120 days, and $>120$ days (Porta et al. 2007); and Mann-Whitney's $U$ test and Student's $t$ test to assess differences between participants with and without available toenails by sociodemographic characteristics. When a trend was observed, the Jonckheere-Terpstra test for trend was used. Spearman's rank correlation coefficient $(\rho)$ was used to evaluate the correlations between the concentrations of trace elements and the time intervals. For the correlations we only considered clinical events that happened previous to or on the day of the nail sample collection. Correlations without the time intervals equal to zero were also calculated. To analyze the relative influence of time intervals on trace concentrations, general linear models were used. Values of trace elements were normalized by natural logarithmic transformation (Camargo et al. 2019; Kleinbaum et al. 1998). Age, gender, tumor stage, and cholestatic syndrome were treated as potential confounding factors. Results were expressed as adjusted geometric means (GMs) with the corresponding 95\% confidence intervals (CIs). The level of statistical significance was set at 0.05 , and all tests are twotailed. Statistical significance, the precision of the estimates, and the magnitude of the associations were all taken into consideration to assess the significance of the results (Kleinbaum et al. 1998; Lash et al. 2021; Amrhein et al. 2019). Based on previous work and hypotheses (Amaral et al. 2012; Porta et al. 2007, 2008, 2009, 2020; Crous-Bou 2009), we analyzed a limited number of predictors and outcomes, many times lower than the millions of SNPs that are analyzed in an agnostic GWAS, for instance. Furthermore, techniques to adjust for the number of comparisons have been shown to have low efficiency or poor accuracy in studies as ours (Lash et al. 2021). Thus, adjustment for the number of comparisons was not appropriate. Analyses were conducted using SPSS v22 (IBM SPSS Statistics, Armonk, NY, USA, 2013) and Stata 8.0.
Fig. 1 Distributions of the time interval from first symptom of pancreatic cancer to toenails collection, and time interval from first symptom of pancreatic cancer to blood extraction. Blue broken line indicates the median days of the time interval from first symptom of pancreatic cancer to toenails collection (iST), and red broken line indicates the median days of time interval from first symptom to blood extraction (iSB)

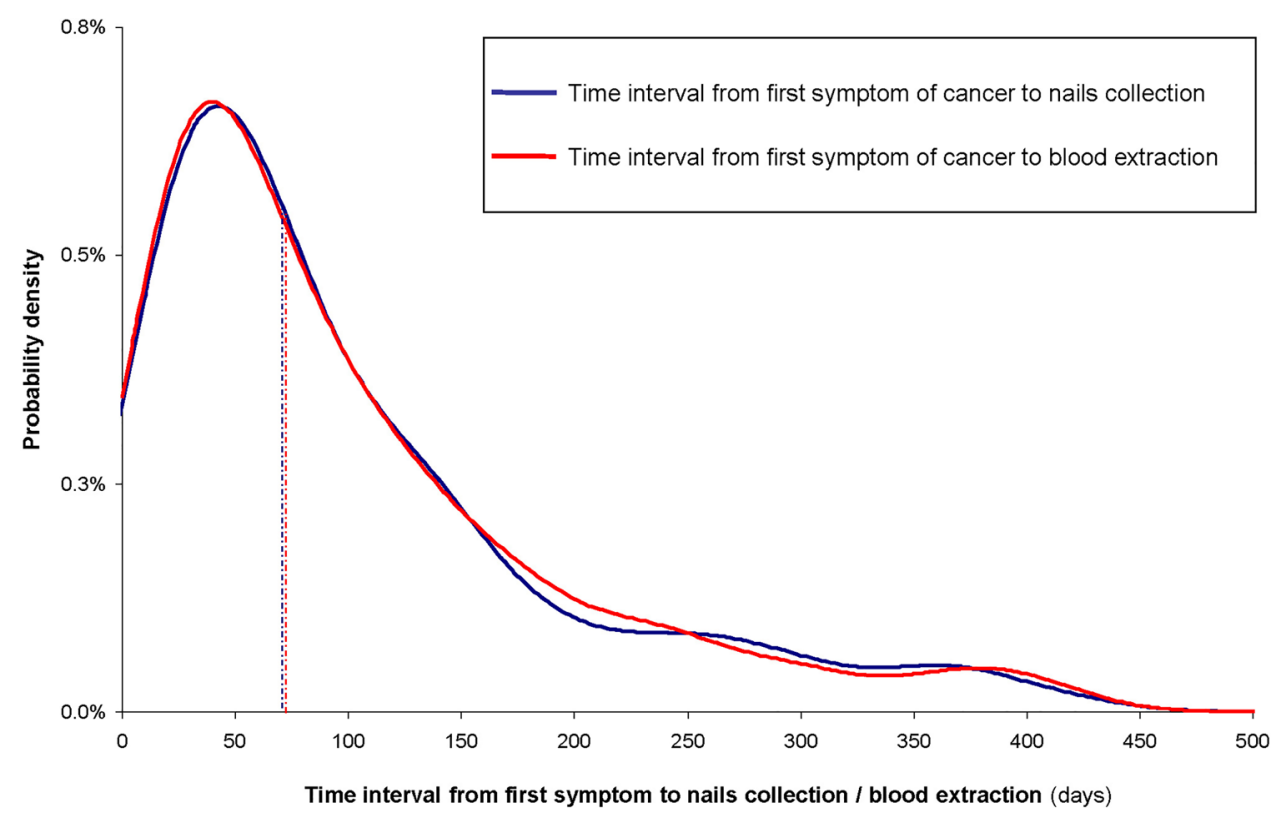




\section{Results}

Median toenail concentrations of the trace elements were weakly or not influenced by iST (Table 1). Crude concentrations of aluminum were slightly higher in subjects with a longer iST: the maximum difference between the medians shown in Table 1 was $5.4 \mu \mathrm{g} / \mathrm{g}$. Concentrations of zinc and selenium were slightly lower in subjects with a longer iST: the maximum difference between
Table 1 Metal concentrations by time from first symptom of pancreatic adenocarcinoma to toenail collection

\begin{tabular}{|c|c|c|c|c|c|c|c|}
\hline \multirow{3}{*}{$\begin{array}{l}\text { Metal concentrations } \\
(\mu \mathrm{g} / \mathrm{g})\end{array}$} & \multicolumn{7}{|c|}{ Interval from first symptom to toenails collection } \\
\hline & \multicolumn{2}{|c|}{$\leq 40$ days } & \multicolumn{2}{|c|}{ 41-120 days } & \multicolumn{2}{|c|}{$>120$ days } & \multirow[b]{2}{*}{$p$ value $^{\mathrm{a}}$} \\
\hline & $N$ & $(\%)$ & $N$ & $(\%)$ & $N$ & $(\%)$ & \\
\hline Number of subjects & 36 & $(30.5)$ & 45 & $(38.1)$ & 37 & $(31.4)$ & \\
\hline \multicolumn{8}{|l|}{ Aluminum } \\
\hline GM $(95 \% \mathrm{CI})$ & 8.02 & $(6.1-10.6)$ & 9.27 & $(7.2-11.9)$ & 11.73 & $(8.9-15.5)$ & 0.159 \\
\hline Median & & 6.96 & & 8.62 & & 12.35 & $0.080^{\mathrm{b}}$ \\
\hline \multicolumn{8}{|l|}{ Nickel } \\
\hline GM $(95 \% \mathrm{CI})$ & 0.22 & $(0.16-0.32)$ & 0.25 & $(0.18-0.34)$ & 0.24 & $(0.17-0.34)$ & 0.929 \\
\hline Median & & 0.23 & & 0.24 & & 0.22 & $0.773^{\mathrm{c}}$ \\
\hline \multicolumn{8}{|l|}{ Zinc } \\
\hline GM $(95 \% \mathrm{CI})$ & 124.8 & $(114-137)$ & 113.5 & $(104-123)$ & 108.0 & $(98.5-118)$ & 0.085 \\
\hline Median & & 115.0 & & 107.1 & & 106.8 & $0.023^{\mathrm{b}}$ \\
\hline \multicolumn{8}{|l|}{ Arsenic } \\
\hline GM $(95 \% \mathrm{CI})$ & 0.07 & $(0.06-0.09)$ & 0.08 & $(0.07-0.10)$ & 0.07 & $(0.06-0.09)$ & 0.525 \\
\hline Median & & 0.07 & & 0.08 & & 0.06 & $0.435^{\mathrm{c}}$ \\
\hline \multicolumn{8}{|l|}{ Selenium } \\
\hline GM $(95 \% \mathrm{CI})$ & 0.54 & $(0.49-0.59)$ & 0.51 & $(0.47-0.55)$ & 0.46 & $(0.42-0.50)$ & 0.039 \\
\hline Median & & 0.54 & & 0.51 & & 0.50 & $0.055^{\mathrm{b}}$ \\
\hline \multicolumn{8}{|l|}{ Cadmium } \\
\hline GM $(95 \%$ CI $)$ & 0.03 & $(0.02-0.05)$ & 0.02 & $(0.01-0.04)$ & 0.03 & $(0.02-0.05)$ & 0.677 \\
\hline Median & & 0.03 & & 0.03 & & 0.03 & $0.812^{\mathrm{c}}$ \\
\hline \multicolumn{8}{|l|}{ Lead } \\
\hline GM $(95 \% \mathrm{CI})$ & 0.87 & $(0.61-1.24)$ & 0.86 & $(0.63-1.18)$ & 0.89 & $(0.63-1.26)$ & 0.986 \\
\hline Median & & 0.80 & & 0.91 & & 0.86 & $0.899^{c}$ \\
\hline \multicolumn{8}{|l|}{ Vanadium } \\
\hline GM $(95 \%$ CI $)$ & 0.02 & $(0.02-0.03)$ & 0.02 & $(0.02-0.03)$ & 0.03 & $(0.02-0.03)$ & 0.753 \\
\hline Median & & 0.02 & & 0.02 & & 0.02 & $0.761^{\mathrm{c}}$ \\
\hline \multicolumn{8}{|l|}{ Chromium } \\
\hline GM $(95 \% \mathrm{CI})$ & 0.37 & $(0.25-0.54)$ & 0.54 & $(0.38-0.76)$ & 0.49 & $(0.34-0.72)$ & 0.306 \\
\hline Median & & 0.33 & & 0.52 & & 0.59 & $0.240^{\mathrm{b}}$ \\
\hline \multicolumn{8}{|l|}{ Manganese } \\
\hline GM $(95 \%$ CI $)$ & 0.29 & $(0.20-0.40)$ & 0.23 & $(0.17-0.32)$ & 0.27 & $(0.19-0.37)$ & 0.679 \\
\hline Median & & 0.27 & & 0.21 & & 0.22 & $0.634^{\mathrm{c}}$ \\
\hline \multicolumn{8}{|l|}{ Iron } \\
\hline GM $(95 \% \mathrm{CI})$ & 16.41 & $(12.4-21.7)$ & 15.30 & (11.9-19.7) & 17.91 & (13.6-23.6) & 0.707 \\
\hline Median & & 14.88 & & 11.49 & & 12.68 & $0.443^{\mathrm{c}}$ \\
\hline \multicolumn{8}{|l|}{ Copper } \\
\hline GM $(95 \% \mathrm{CI})$ & 3.81 & $(3.3-4.4)$ & 3.65 & $(3.2-4.1)$ & 3.69 & $(3.2-4.2)$ & 0.892 \\
\hline Median & & 3.36 & & 3.53 & & 3.50 & $0.915^{\mathrm{c}}$ \\
\hline
\end{tabular}

Total number of subjects: $118, G M$ geometric mean, $C I$ confidence interval

${ }^{a}$ Unless otherwise specified, $p$ value derived from ANOVA

${ }^{\mathrm{b}}$ Jonckheere-Terpstra test for trend

${ }^{c}$ Kruskal-Wallis test (two-tailed) 
the same medians (Table 1) was $8.2 \mu \mathrm{g} / \mathrm{g}$ and $0.04 \mu \mathrm{g} / \mathrm{g}$, respectively. The cloud of data points shows how weak the relationship with zinc is (Fig. 2), and so does the $R^{2}$ : only $1.5 \%$ of the variability in concentrations of zinc is statistically explained by the iST.

After adjustment for age and sex, concentrations of aluminum continued to increase weakly with iST (maximum difference, $3.7 \mu \mathrm{g} / \mathrm{g}$ ) (Table 2, Model 1). When models were further adjusted for tumor stage (Model 2) and/or the cholestatic syndrome (Table 3, Models 3 and 4), results for aluminum did not materially change.

For zinc and selenium, the inverse relations observed with iST became slightly stronger after adjustment for age, sex, cholestatic syndrome, and tumor stage (Tables 2 and 3). The difference between the fully adjusted GM of zinc concentrations for iST $\leq 40$ days and iST $>120$ days was of $20.4 \mu \mathrm{g} / \mathrm{g}$ (GMs $=127.1$ and $106.7 \mu \mathrm{g} / \mathrm{g}$, respectively) (Table 3, Model 4). In the fully adjusted models, zinc concentrations were inversely associated also with the cholestatic syndrome and tumor stage; i.e., patients with partial or complete cholestatic syndrome, and patients in more advanced tumor stages had lower zinc toenail concentrations than patients with no cholestatic syndrome or the tumor at stage I (differences of about $20 \mu \mathrm{g} / \mathrm{g}$ or less) (Table 3, Model 4). Cholestatic syndrome and tumor stage were not associated with concentrations of the other elements analyzed.

Age and sex-adjusted concentrations of selenium, as well as concentrations of selenium further adjusted for tumor stage, decreased weakly with increasing iST (difference of $0.08 \mu \mathrm{g} / \mathrm{g}$ for iST $>120$ days vs. $\leq 40$ days) (Table 2);

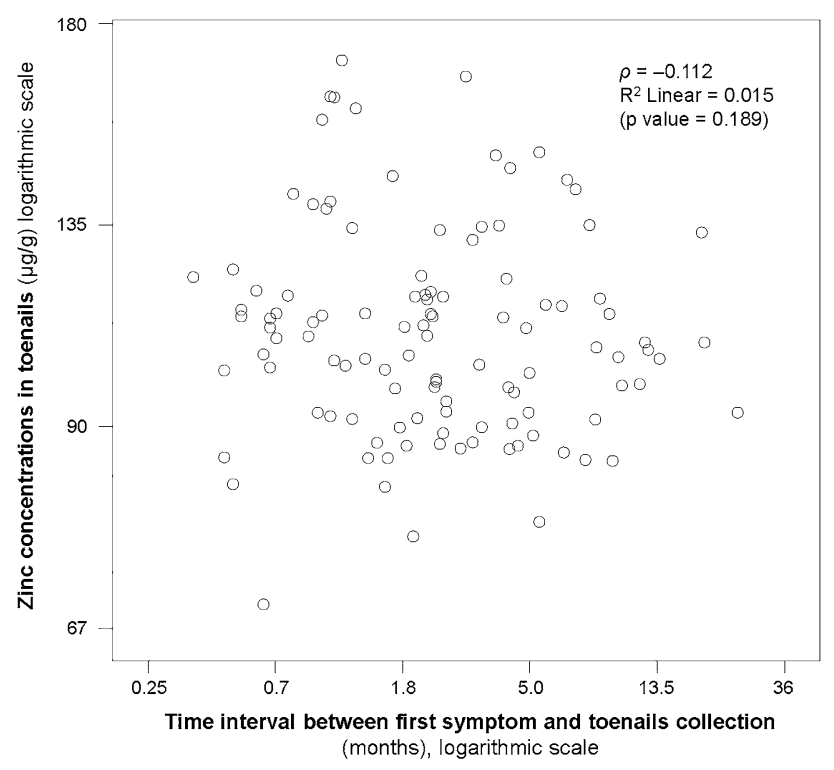

Fig. 2 Scatterplot of zinc concentrations against the time interval from first symptom of pancreatic cancer to toenails collection. $\rho$ : Spearman's rho coefficient selenium concentrations were not influenced by stage nor by cholestatic syndrome (Table 3 ).

Virtually all correlations between time intervals and metal concentrations were modest, with all Spearman's $\rho<0.35$ (from -0.33 to 0.32 ) (Table 4). Concerning toenail levels of aluminum, future studies may consider their associations with iST $(\rho=0.19)$, with the interval from diagnosis to toenail collection $(\rho=0.25)$, and with the interval from the exploratory laparotomy to toenail collection $(\rho=0.31)$; in the analysis of these correlations we took into account only clinical events that took place before or the same day as toenail collection. Also worth noting were the associations between concentrations of iron and the interval from diagnosis to toenail collection $(\rho=0.29)$, and between cadmium levels and the interval from treatment onset to toenail collection $(\rho=-0.33)$ (Table 4).

\section{Discussion}

Toenail concentrations of the 12 trace elements studied were weakly or not influenced by the progression of the disease or the diagnostic process. Levels of aluminum increased slightly with increasing iST, whereas levels of zinc and selenium decreased weakly.

Adjustment for multiple factors did not materially change the results. Thus, when adjusting for age and sex, concentrations of aluminum continued to increase weakly and statistically non-significantly with iST. Further adjusting for tumor stage or cholestatic syndrome (Porta et al. 2009, 2005) did not change these findings. For zinc and selenium, the inverse relation observed with iST became slightly stronger after further adjusting for cholestatic syndrome, and tumor stage. Moreover, mutually adjusted models showed that all iST, cholestatic syndrome, and stage were weakly but statistically significantly related to levels of zinc; the relations with cholestatic syndrome and tumor stage were not observed for selenium or the other trace elements. Thus, for instance, future studies could confirm whether at diagnosis concentrations of zinc are lower in patients with more disseminated than with more localized tumors. It remains to be seen whether the weak magnitude of the changes in concentrations that we observed (about $20 \mu \mathrm{g} / \mathrm{g}$ or less) is applicable to other studies; if it is, it might not be efficient to measure the chronology of symptoms as comprehensively as we did (Porta et al. 2005), and simpler alternatives could be considered (Fernandez et al. 2002; Porta et al. 1996, 2003b). It may be more feasible to measure intervals between other clinical events (such as diagnosis, clinical procedures as laparotomy, treatment onset) and toenail collection. For future studies, our findings warrant checking the possible effects of disease progression on toenail levels of at least aluminum, zinc, and selenium. Findings also suggest that, with the mentioned 
Table 2 Influence of the interval from first symptom of pancreatic adenocarcinoma to toenail collection (iST) on concentrations of trace elements $(\mu \mathrm{g} / \mathrm{g})$

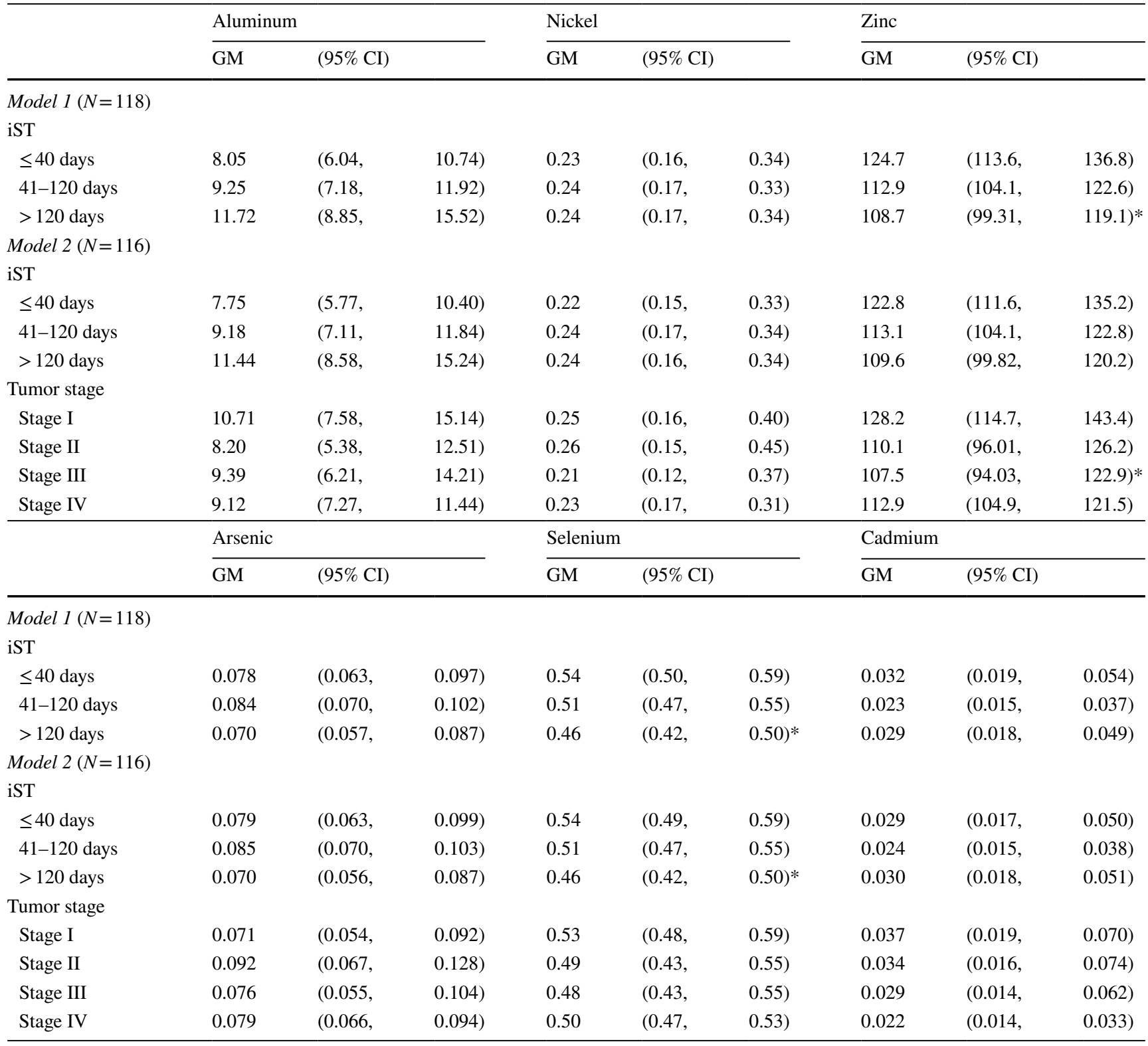

All models are adjusted for age and sex. Furthermore, in Model 2 iST and stage are also mutually adjusted

$i S T$ interval from first symptom to toenails collection, $G M$ geometric mean, $C I$ confidence interval

$* p$ value $<0.05$ (vs. iST $\leq 40$ days or vs. tumor stage I)

checks, it may be valid to use toenail samples collected at diagnosis.

We previously reported that pancreatic cancer patients with cholestatic syndrome had significantly lower serum concentrations of OCs than patients with other signs and symptoms (Porta et al. 2009). When symptoms were taken into account, tumor stage had only weak (and inverse) relationships with all OCs. Overall, the prevailing direction was for most signs and symptoms to lower serum OCs concentrations, even when the latter were lipid-corrected (Porta et al. 2008). These relationships with symptoms were not observed in the present study with trace elements measured in toenails; most likely, because concentrations of such biomarkers, which are not lipophilic, are not influenced by the pathophysiological processes inherent to the progression of pancreatic cancer (Agency for Toxic Substances and Disease Registry (ATSDR). Toxicological Profiles. U.S. Department of Health and Human Services).

Variations in concentrations of aluminum according to iST suggest that accumulation of this element in 
Table 3 Influence of time interval from first symptom to toenails collection (iST) on toenail concentrations of metals ( $\mu \mathrm{g} / \mathrm{g}$ )

\begin{tabular}{|c|c|c|c|c|c|c|c|c|c|}
\hline & \multicolumn{3}{|c|}{ Aluminum } & \multicolumn{3}{|c|}{ Nickel } & \multicolumn{3}{|l|}{ Zinc } \\
\hline & GM & \multicolumn{2}{|l|}{$(95 \% \mathrm{CI})$} & GM & \multicolumn{2}{|c|}{$(95 \% \mathrm{CI})$} & GM & \multicolumn{2}{|l|}{$(95 \% \mathrm{CI})$} \\
\hline \multicolumn{10}{|l|}{ Model $3(N=118)$} \\
\hline \multicolumn{10}{|l|}{ iST $^{a}$} \\
\hline$\leq 40$ days & 8.08 & $(5.95$ & 10.99) & 0.24 & $(0.16$ & $0.35)$ & 127.9 & $(116.0$ & 140.9) \\
\hline 41-120 days & 9.21 & (7.13, & 11.90) & 0.24 & $(0.17$ & $0.33)$ & 112.7 & $(103.9$ & 122.2) \\
\hline$>120$ days & 11.74 & $(8.77$ & 15.73) & 0.24 & $(0.16$ & $0.34)$ & 106.4 & (97.01, & $116.7)^{*}$ \\
\hline \multicolumn{10}{|l|}{ Cholestatic syndrome ${ }^{\mathrm{b}}$} \\
\hline No syndrome & 9.12 & $(6.72$ & 12.38) & 0.24 & $(0.17$ & $0.36)$ & 126.1 & $(114.4$ & 138.9) \\
\hline Partial syndrome & 10.82 & $(7.83$ & $14.95)$ & 0.23 & $(0.15$ & $0.35)$ & 107.8 & $(97.30$ & $119.4)^{*}$ \\
\hline Complete syndrome & 9.23 & $(7.25$ & $11.75)$ & 0.24 & $(0.17$ & $0.32)$ & 112.1 & $(103.9$, & 121.1) \\
\hline \multicolumn{10}{|l|}{ Model $4(N=116)$} \\
\hline \multicolumn{10}{|l|}{ iST $^{a}$} \\
\hline$\leq 40$ days & 8.00 & $(5.84$ & $10.95)$ & 0.22 & $(0.15$ & $0.34)$ & 127.1 & (115.1, & 140.3) \\
\hline 41-120 days & 9.07 & (7.01, & 11.74) & 0.24 & $(0.17$ & $0.34)$ & 112.4 & (103.7, & 121.9) \\
\hline$>120$ days & 11.25 & $(8.36$ & 15.14) & 0.24 & $(0.16$ & $0.35)$ & 106.7 & (97.23, & $117.2)^{*}$ \\
\hline \multicolumn{10}{|l|}{ Cholestatic syndrome $^{\mathrm{b}}$} \\
\hline No syndrome & 9.55 & $(6.96$ & 13.11) & 0.24 & $(0.16$ & $0.37)$ & 129.1 & $(116.8$ & 142.6) \\
\hline Partial syndrome & 10.63 & (7.64, & $14.80)$ & 0.22 & $(0.14$ & $0.34)$ & 108.6 & $(97.86$ & $120.4)^{*}$ \\
\hline Complete syndrome & 8.62 & $(6.68$ & 11.12) & 0.24 & $(0.17$ & $0.33)$ & 109.4 & $(101.0$ & $118.5)^{*}$ \\
\hline \multicolumn{10}{|l|}{ Tumor stage $^{\mathrm{c}}$} \\
\hline Stage I & 11.01 & (7.70, & $15.75)$ & 0.25 & $(0.16$ & $0.40)$ & 131.5 & $(117.5$, & 147.1) \\
\hline Stage II & 8.06 & $(5.25$ & 12.37) & 0.26 & $(0.15$ & $0.45)$ & 108.1 & $(94.49$ & $123.7)^{*}$ \\
\hline Stage III & 9.60 & $(6.28$ & 14.66) & 0.22 & $(0.12$ & $0.38)$ & 110.8 & $(96.96$ & $126.6)^{*}$ \\
\hline \multirow[t]{3}{*}{ Stage IV } & 9.00 & $(7.14$ & $11.35)$ & 0.23 & $(0.17$ & $0.31)$ & 111.3 & $(103.5$ & $119.7)^{*}$ \\
\hline & \multicolumn{3}{|c|}{ Arsenic } & \multicolumn{3}{|c|}{ Selenium } & \multicolumn{3}{|c|}{ Cadmium } \\
\hline & GM & $(95 \% \mathrm{CI})$ & & GM & $(95 \% \mathrm{CI}$ & & GM & $(95 \% \mathrm{CI})$ & \\
\hline \multicolumn{10}{|l|}{ Model $3(N=118)$} \\
\hline \multicolumn{10}{|l|}{$\mathrm{iST}^{\mathrm{a}}$} \\
\hline$\leq 40$ days & 0.082 & $(0.065$ & $0.103)$ & 0.55 & $(0.50$ & $0.61)$ & 0.035 & $(0.020$ & $0.061)$ \\
\hline $41-120$ days & 0.083 & $(0.068$ & $0.101)$ & 0.51 & $(0.47$ & $0.55)$ & 0.022 & $(0.014$ & $0.036)$ \\
\hline$>120$ days & 0.068 & $(0.055$ & $0.085)$ & 0.45 & $(0.41$ & $0.49)^{*}$ & 0.028 & $(0.016$ & $0.047)$ \\
\hline Cholestatic syndrome $^{\mathrm{b}}$ & & & & & & & & & \\
\hline No syndrome & 0.084 & $(0.067$ & $0.106)$ & 0.54 & $(0.49$ & $0.59)$ & 0.029 & $(0.017$ & $0.049)$ \\
\hline Partial syndrome & 0.083 & $(0.065$ & $0.106)$ & 0.48 & $(0.44$ & $0.53)$ & 0.043 & $(0.024$ & $0.077)$ \\
\hline Complete syndrome & 0.072 & $(0.060$ & $0.086)$ & 0.49 & $(0.46$ & $0.53)$ & 0.021 & $(0.014$ & $0.033)$ \\
\hline Model $4(N=116)$ & & & & & & & & & \\
\hline iST $^{\mathrm{a}}$ & & & & & & & & & \\
\hline$\leq 40$ days & 0.082 & $(0.064$ & $0.104)$ & 0.56 & $(0.51$ & $0.61)$ & 0.034 & $(0.019$ & $0.060)$ \\
\hline 41-120 days & 0.084 & $(0.069$ & $0.103)$ & 0.51 & $(0.47$ & $0.55)$ & 0.023 & $(0.014$ & $0.036)$ \\
\hline$>120$ days & 0.068 & $(0.054$ & $0.086)$ & 0.45 & $(0.41$ & $0.49)^{*}$ & 0.028 & $(0.016$ & $0.048)$ \\
\hline Cholestatic syndrome $^{\mathrm{b}}$ & & & & & & & & & \\
\hline No syndrome & 0.083 & $(0.065$ & $0.106)$ & 0.55 & $(0.50$ & $0.60)$ & 0.032 & $(0.018$ & $0.057)$ \\
\hline Partial syndrome & 0.083 & $(0.064$ & $0.107)$ & 0.49 & $(0.44$ & $0.54)$ & 0.045 & $(0.025$ & $0.081)$ \\
\hline Complete syndrome & 0.073 & $(0.060$ & $0.089)$ & 0.48 & $(0.44$ & $0.52)$ & 0.019 & $(0.012$ & $0.030)$ \\
\hline Tumor stage $^{c}$ & & & & & & & & & \\
\hline Stage I & 0.072 & $(0.055$ & $0.096)$ & 0.54 & $(0.49$ & $0.61)$ & 0.042 & $(0.022$ & $0.080)$ \\
\hline Stage II & 0.091 & $(0.065$ & $0.127)$ & 0.48 & $(0.42$ & $0.54)$ & 0.031 & $(0.014$ & $0.068)$ \\
\hline Stage III & 0.078 & $(0.056$ & $0.108)$ & 0.50 & $(0.44$ & $0.57)$ & 0.032 & $(0.015$ & $0.070)$ \\
\hline Stage IV & 0.077 & $(0.065$ & $0.093)$ & 0.49 & $(0.46$ & $0.53)$ & 0.021 & $(0.014$ & $0.031)$ \\
\hline
\end{tabular}


Table 3 (continued)

\author{
All models are adjusted for age and sex \\ $i S T$ interval from first symptom to toenails collection, $G M$ geometric mean, $C I$ confidence interval \\ ${ }^{\text {a }}$ Reference category is $\leq 40$ days of interval \\ ${ }^{\mathrm{b}}$ Reference category is no cholestatic syndrome \\ ${ }^{\mathrm{c}}$ Reference category is tumor stage I \\ $* p$ value $<0.05$
}

Table 4 Spearman's correlations $(\rho)$ between concentrations of trace elements $(\mu \mathrm{g} / \mathrm{g}$ ) and time intervals (days) from clinical events to collection of toenails

\begin{tabular}{|c|c|c|c|c|c|c|c|c|c|c|}
\hline & \multicolumn{10}{|c|}{ Clinical events related to pancreatic cancer } \\
\hline & $\begin{array}{l}\text { First } \\
\text { symptom }\end{array}$ & $\begin{array}{l}\text { Hospital } \\
\text { admission }\end{array}$ & Interview & Diagnosis & $\begin{array}{l}\text { Treatment } \\
\text { onset }\end{array}$ & $\begin{array}{l}\text { Ultrasound } \\
\text { scan }\end{array}$ & $\mathrm{CT}$ & Fibrogastroscopy & ERCP & $\begin{array}{l}\text { Exploratory } \\
\text { laparotomy }\end{array}$ \\
\hline & $N(\%)$ & $N(\%)$ & $N(\%)$ & $N(\%)$ & $N(\%)$ & $N(\%)$ & $N(\%)$ & $N(\%)$ & $N(\%)$ & $N(\%)$ \\
\hline Total number & $118(100)$ & $115(97.5)$ & $97(82.2)$ & $68(57.6)$ & $36(30.5)$ & $101(85.6)$ & $82(69.5)$ & $30(25.4)$ & $41(34.7)$ & $37(31.4)$ \\
\hline Aluminum & $0.186^{* *}$ & 0.087 & -0.121 & $0.253 * *$ & 0.090 & 0.063 & 0.089 & -0.144 & 0.054 & $0.311^{*}$ \\
\hline Nickel & 0.001 & 0.082 & -0.109 & 0.201 & -0.009 & 0.138 & 0.162 & 0.052 & 0.207 & 0.143 \\
\hline Zinc & -0.112 & -0.017 & -0.020 & -0.075 & -0.216 & 0.084 & -0.025 & 0.084 & 0.043 & -0.092 \\
\hline Arsenic & -0.032 & 0.084 & -0.033 & 0.022 & -0.102 & 0.048 & 0.129 & $0.321^{*}$ & -0.006 & -0.152 \\
\hline Selenium & -0.117 & -0.002 & 0.089 & 0.062 & 0.026 & 0.120 & 0.124 & -0.014 & 0.045 & -0.028 \\
\hline Cadmium & -0.012 & 0.061 & -0.159 & -0.063 & $-0.327^{*}$ & -0.074 & -0.020 & -0.110 & -0.129 & -0.166 \\
\hline Lead & -0.001 & 0.073 & -0.128 & 0.049 & -0.247 & 0.041 & 0.144 & 0.073 & -0.080 & -0.061 \\
\hline Vanadium & 0.079 & 0.005 & -0.128 & 0.125 & -0.051 & 0.013 & 0.059 & -0.192 & -0.072 & 0.062 \\
\hline Chromium & 0.065 & 0.013 & 0.097 & 0.019 & -0.064 & 0.030 & 0.094 & -0.094 & -0.071 & 0.003 \\
\hline Manganese & -0.061 & -0.045 & -0.131 & 0.092 & -0.225 & -0.096 & -0.078 & -0.225 & -0.130 & -0.108 \\
\hline Iron & 0.010 & -0.052 & -0.143 & $0.292 * *$ & 0.084 & 0.028 & -0.041 & -0.038 & -0.098 & -0.171 \\
\hline Copper & 0.054 & 0.046 & -0.058 & 0.121 & -0.004 & 0.139 & -0.069 & 0.149 & 0.104 & -0.054 \\
\hline
\end{tabular}

Total number of subjects: 118

$C T$ computerized axial tomography, ERCP endoscopic retrograde cholangiopancreatography

$* 0.05<p$ value $\leq 0.10$

$* * p$ value $\leq 0.05$

pancreatic cancer patients could be enhanced, maybe due to an increased intestinal absorption and decreased excretion of the metal (Igbokwe et al. 2019). Lower toenail concentrations of selenium and zinc during the development of pancreatic cancer could be due to nutritional needs of the tumor itself.

Disease progression bias (Porta et al. 2014) may occur when the biological samples where the exposure biomarkers will be analyzed are collected just before or after the diagnosis of the disease, and when disease-related conditions experienced during the development of the disease and around its diagnosis cause a change in the biomarkers. One type of such conditions are the disease-induced pathophysiological processes that we already mentioned in the Introduction and in previous papers (Porta et al. 2007, 2008, 2009, 2014,
2005, 2021; Porta 2001; Gasull et al. 2019). Related but different are the metabolic requirements of the tumor $(\mathrm{He} 2011$; Gutiérrez-González et al. 2019; Salcedo-Bellido et al. 2021; Signes-Pastor et al. 2020; Longnecker et al. 1993; Garland et al. 1993; Hunter et al. 1990; Hopps 1977; Sukumar 2006). A third mechanism can operate as follows: symptoms (such as tiredness, loss of appetite, weight loss, abdominal pain, nausea) caused by the still subclinical disease can cause changes in the diet of the subject that change the intake of some nutrients and subsequently their body concentrations. Naturally, other changes in behaviors promoted by the clinically emerging but yet undiagnosed disease can affect other lifestyle factors (e.g., smoking) and the corresponding exposures. The common effect of all these processes is that the disease-altered exposure biomarkers measured around the 
time of diagnosis lack etiologic significance: they do not reflect the causally relevant exposures that took place in the more distant past (Porta et al. 2014).

In a previous case-control study we found that individuals with the lowest levels of selenium in toenails at diagnosis presented a higher risk of pancreatic cancer (Amaral et al. 2012). Also, lower levels of zinc were associated with a higher risk of a KRAS mutated pancreatic cancer (GómezTomás et al. 2019). In the present study, the inverse associations of the concentrations of zinc and selenium with iST were not explained by tumor stage. Thus, the development of the disease (Porta et al. 2014) could partly explain the lower levels of selenium observed in the case-control study (Amaral et al. 2012).

Concentrations of the other trace elements analyzed were not associated with time intervals from clinical events to toenails collection. Such elements include cadmium, lead, and arsenic, the three elements previously found positively associated with pancreatic cancer risk (Amaral et al. 2012). Thus, even in a biologically and clinically aggressive disease as pancreatic cancer, toenail concentrations of trace elements may provide a measure of mid-term intake of trace elements unaffected by clinical events and disease progression.

In our study participants the toenail concentrations of aluminium, selenium, zinc and the other elements were similar to concentrations in the homogenized published literature in the same matrix (Amaral et al. 2012; Gutiérrez-González et al. 2019; Salcedo-Bellido et al. 2021; Signes-Pastor et al. 2020).

Problems in using toenails as a matrix for measurement of biomarkers include variability in growth rate, external contamination, and inconsistent protocols for collection and analysis (He 2011; Gutiérrez-González et al. 2019; SalcedoBellido et al. 2021; Signes-Pastor et al. 2020; Orloff et al. 2009). Because in the present study sample collection was conducted in-hospital, collection of toenails was similar for all study patients, and the external contamination of nails was minimized by having toenails collected immediately after bathing and scraping, and by sonicating nails in the laboratory prior to analysis (Nichols et al. 1998). All nails were analyzed simultaneously in a laboratory with state-of-the art procedures (Gómez-Tomás et al. 2019; Camargo et al. 2019; Amaral et al. 2012; Fleckman 1985). In some instances a small SRM sample mass may have been the cause of some of the variability seen in the within- and between-batch SRM results (Supplementary Table 2).

In our study, patients were screened for eligibility early during the hospital admission, and selected, if eligible (Camargo et al. 2019). Epidemiological studies of risk factors rarely include information on the health care process that patients undergo before inclusion. In some health care systems, computerized medical records shared by primary health care centers and hospitals include information on the duration of symptoms, clinical procedures, and disease progression (Porta et al. 2009, 1996; Fernandez et al. 2002; Gavaldà et al. 1995). Our approach integrates concepts and methods from clinical, environmental, and molecular epidemiology (Gallo et al. 2011).

Limitations of our study include its relatively small sample size, and unmeasured factors potentially related to concentrations of trace elements, such as, perhaps, body mass index (BMI) (He 2011), and, likely, size of the toe; however, our clippings were from the larger toe, all sample preparation steps were recorded gravimetrically, and state-of-the-art methods were used in a reference laboratory. Signs and especially symptoms are by nature difficult to measure, although our methods on these aspects are among the most reliable (Porta et al. 2005, 1996, 2003b; Fernandez et al. 2002), and this information is unlikely to be available from other studies. The present study did not aim at identifying the dietary or environmental sources of the trace elements found.

Repeated toenail samples collected during years from individual cohort members would allow to more directly assess individual longitudinal changes over time (even before the subclinical onset of the disease), and intraindividual variability (He 2011), but such samples are seldom available in large studies. The aims and methodological frameworks that are relevant to assess reproducibility over time of measurements of metals in toenails and other matrixes in healthy and stable individuals (He 2011) are different from ours (Porta et al. 2007, 2014; Porta 2001) (see Introduction); notably, the former rarely consider the influence of the subclinical or clinical disease of interest; they may also lack valid data on other relevant changes (BMI, lifestyles, environmental and social conditions). Nevertheless, there are obvious relationships.

As expected (Porta et al. 2009; Porta 2001), a substantial number of clinical and epidemiological studies continue to be based on diagnosed cases; hence, they collect post-diagnostic biological samples from cases, rather than collecting the samples from the entire cohort at baseline and conducting nested case-control studies after cases are ascertained during follow-up (Gasull et al. 2019; Porta et al. 2021). Among other strengths, the latter approach has considerable advantages with regard to timing of the biological sample collection; notably, avoidance of disease progression bias.

Our original approach and findings may be of use when designing and monitoring the conduct of studies (e.g., intervals between first symptom of the disease, first contact with carers or first hospital admission and collection of toenails, diagnostic procedures performed before nails collection). They also have implications for the analysis and interpretation of future studies of pancreatic cancer and possibly other cancers. The findings warrant checking the possible effects of disease progression on toenail levels of at least aluminum, zinc, and selenium. Findings also suggest that 
with the mentioned checks it may be valid to measure trace elements in toenail samples collected at diagnosis, not necessarily years before diagnosis.

Supplementary Information The online version contains supplementary material available at https://doi.org/10.1007/s12403-021-00436-2.

Acknowledgements The authors gratefully acknowledge scientific advice provided by Núria Malats, Paco Real, and André F. S. Amaral. Technical assistance from Ana Alfaro, Tomàs López, Andrea Sánchez Rodríguez, Natalia Pallarès, Mirari Márquez, Yolanda Rovira, and Meri Corominas is also gratefully acknowledged.

Funding The work was supported in part by research grants from Instituto de Salud Carlos III-FEDER, Ministry of Health, Government of Spain (FIS PI17/0088, FIS PI21/0052, and CIBER de Epidemiología y Salud Pública-CIBERESP); the Hospital del Mar Medical Research Institute (IMIM), Barcelona; Fundació La Marató de TV3 (20132910); the CRUE-Santander Fondo Supera Covid-19 (15072020); the Government of Catalonia (2014 SGR 1012, 2017 SGR 439); the Association for International Cancer Research (AICR09-0780); Fundación Científica de la Asociación Española Contra el Cáncer (AECC); and the Intramural Research Program of the Division of Cancer Epidemiology and Genetics, National Cancer Institute, USA.

Data Availability Not applicable.

Code Availability Not applicable.

\section{Declarations}

Conflict of interest The authors have no conflicts of interest in connection with the paper, and declare no competing financial interests.

Ethical Approval The Ethics Committees of participating hospitals approved the study protocol.

Consent to Participate Patients gave informed consent to be included in the study.

\section{References}

Agency for Toxic Substances and Disease Registry (ATSDR). Toxicological Profiles. U.S. Department of Health and Human Services. Atlanta. https://www.atsdr.cdc.gov/toxprofiledocs/index.html. Accessed 15 Oct 2021

Amaral AF, Porta M, Silverman DT, Milne RL, Kogevinas M, Rothman N et al (2012) Pancreatic cancer risk and levels of trace elements. Gut 61:1583-1588

Amrhein V, Greenland S, McShane B (2019) Scientists rise up against statistical significance. Nature 567:305-307

Armitage P, Berry G, Matthews JNS (2002) Statistical methods in medical research, 4th edn. Blackwell, Oxford

Brocato J, Chen D, Liu J, Fang L, Jin C, Costa M (2015) A potential new mechanism of arsenic carcinogenesis: depletion of stem-loop binding protein and increase in polyadenylated canonical histone h3.1 mrna. Biol Trace Elem Res 166:72-81

Camargo J, Pumarega JA, Alguacil J, Sanz-Gallén P, Gasull M, Delclos GL et al (2019) Toenail concentrations of trace elements and occupational history in pancreatic cancer. Environ Int 127:216-225
Chen QY, Costa M (2017) A comprehensive review of metal-induced cellular transformation studies. Toxicol Appl Pharmacol 331:33-40

Chen C, Xun P, Nishijo M, Sekikawa A, He K (2015) Cadmium exposure and risk of pancreatic cancer: a meta-analysis of prospective cohort studies and case-control studies among individuals without occupational exposure history. Environ Sci Pollut Res Int 22:17465-17474

Chervona Y, Arita A, Costa M (2012) Carcinogenic metals and the epigenome: understanding the effect of nickel, arsenic, and chromium. Metallomics 4:619-627

Crous-Bou M (2009) Clinical and environmental influences on the prevalence of mutations in the K-ras oncogene in patients with pancreatic ductal adenocarcinoma [doctoral dissertation]. Barcelona: Universitat Autònoma de Barcelona. Catalan and English. http://www.imim.es/programesrecerca/epidemiologia/documentsg recm.html. Accessed 15 Oct 2021

Fernandez E, La Vecchia C, Porta M, Negri E, Lucchini F, Levi F (1994) Trends in pancreatic cancer mortality in Europe, 19551989. Int J Cancer 57:786-792

Fernandez E, Porta M, Malats N, Belloc J, Gallén M (2002) Symptom to diagnosis interval and survival in cancers of the digestive tract. Dig Dis Sci 47:2434-2440

Fleckman P (1985) Anatomy and physiology of the nail. Dermatol Clin 3:373-381

Gallo V, Egger M, McCormack V, Farmer PB, Ioannidis JPA, KirschVolders M et al (2011) STrengthening the Reporting of OBservational studies in Epidemiology-Molecular Epidemiology (STROBE-ME): an extension of the STROBE statement. Eur J Epidemiol 26:797-810

Garland M, Morris JS, Rosner BA, Stampfer MJ, Spate VL, Baskett CJ et al (1993) Toenail trace element levels as biomarkers: reproducibility over a 6-year period. Cancer Epidemiol Biomark Prev 2:493-497

Gasull M, Pumarega J, Kiviranta H, Rantakokko P, Raaschou-Nielsen O, Bergdahl IA et al (2019) Methodological issues in a prospective study on plasma concentrations of persistent organic pollutants and pancreatic cancer risk within the EPIC cohort. Environ Res 169:417-433

Gavaldà L, Porta M, Malats N, Piñol JL, Fernández E, Maguire A et al (1995) Agreement between information supplied by the patient and a family member on medical history, consumption of tobacco, alcohol and coffee, and diet in cancer of the exocrine pancreas and extrahepatic biliary tract. Gac Sanit 9:334-342

Gómez-Tomás Á, Pumarega J, Alguacil J, Amaral AFS, Malats N, Pallarès $\mathrm{N}$ et al (2019) Concentrations of trace elements and KRAS mutations in pancreatic ductal adenocarcinoma. Environ Mol Mutagen 60:693-703

Gutiérrez-González E, García-Esquinas E, de Larrea-Baz NF, SalcedoBellido I, Navas-Acien A, Lope V et al (2019) Toenails as biomarkers of exposure to essential trace metals: a review. Environ Res 179(Pt A): 108787

He K (2011) Trace elements in nails as biomarkers in clinical research. Eur J Clin Investig 41:98-102

Hopps HC (1977) The biologic bases for using hair and nail for analyses of trace elements. Sci Total Environ 7:71-89

Huang C, Ke Q, Costa M, Shi X (2004) Molecular mechanisms of arsenic carcinogenesis. Mol Cell Biochem 255:57-66

Huff J, Lunn RM, Waalkes MP, Tomatis L, Infante PF (2007) Cadmium-induced cancers in animals and in humans. Int J Occup Environ Health 13:202-212

Hunter DJ, Morris JS, Chute CG, Kushner E, Colditz GA, Stampfer MJ et al (1990) Predictors of selenium concentration in human toenails. Am J Epidemiol 132:114-122

Igbokwe IO, Igwenagu E, Igbokwe NA (2019) Aluminium toxicosis: a review of toxic actions and effects. Interdiscip Toxicol 12:45-70 
Kleinbaum DG, Kupper LL, Muller KE, Nizam A (1998) Applied regression analysis and other multivariable methods, 3rd edn. Duxbury, Pacific Grove

Koedrith P, Kim HL, Il Weon J, Seo YR (2013) Toxicogenomic approaches for understanding molecular mechanisms of heavy metal mutagenicity and carcinogenicity. Int J Hyg Environ Health 216:587-598

Lash TL, VanderWeele TJ, Haneuse S, Rothman KJ (eds) (2021) Modern epidemiology, 4th edn. Walters-Kluwer, Philadelphia

Longnecker MP, Stampfer MJ, Morris JS, Spate V, Baskett C, Mason M et al (1993) A 1-y trial of the effect of high selenium bread on selenium concentrations in blood and toenails. Am J Clin Nutr 57:408-413

Marchiset-Ferlay N, Savanovitch C, Sauvant-Rochat M (2012) What is the best biomarker to assess arsenic exposure via drinking water? Environ Int 39:150-171

Nichols T, Morris J, Mason M, Spate V, Baskett C, Cheng T et al (1998) The study of human nails as an intake monitor for arsenic using neutron activation analysis. J Radioanal Nucl 236:51-57

Ntihabose R, Surette C, Foucher D, Clarisse O, Bouchard M (2018) Assessment of saliva, hair and toenails as biomarkers of low level exposure to manganese from drinking water in children. Neurotoxicol 64:126-133

Ojajärvi IA, Partanen TJ, Ahlbom A, Boffetta P, Hakulinen T, Jourenkova $\mathrm{N}$ et al (2000) Occupational exposures and pancreatic cancer: a meta-analysis. Occup Environ Med 57:316-324

Orloff K, Mistry K, Metcalf S (2009) Biomonitoring for environmental exposures to arsenic. J Toxicol Environ Health B Crit Rev 12:509-524

Porta M (2001) Role of organochlorine compounds in the etiology of pancreatic cancer: a proposal to develop methodological standards. Epidemiology 12:272-276

Porta M, Gallén M, Belloc J, Malats N (1996) Predictors of the interval between onset of symptoms and first medical visit in patients with digestive tract cancer. Int J Oncol 8:941-949

Porta M, Malats N, Jariod M, Grimalt JO, Rifà J, Carrato A et al (1999) Serum concentrations of organochlorine compounds and K-ras mutations in exocrine pancreatic cancer. Lancet 354:2125-2129

Porta M, Costafreda S, Malats N, Guarner L, Soler M, Gubern JM et al (2000) Validity of the hospital discharge diagnosis in epidemiologic studies of biliopancreatic pathology. Eur J Epidemiol 16:533-541

Porta M, Ayude D, Alguacil J, Jariod M (2003a) Exploring environmental causes of altered ras effects: fragmentation plus integration? Mol Carcinog 36:45-52

Porta M, Fernandez E, Alguacil J (2003b) Semiology, proteomics and the early detection of symptomatic cancer. J Clin Epidemiol $56: 815-819$
Porta M, Fabregat X, Malats N, Guarner L, Carrato A, de Miguel A et al (2005) Exocrine pancreatic cancer: symptoms at presentation and their relation to tumour site and stage. Clin Transl Oncol 7:189-197

Porta M, Pumarega J, Ferrer-Armengou O, López T, Alguacil J, Malats $\mathrm{N}$ et al (2007) Timing of blood extraction in epidemiologic and proteomic studies: Results and proposals from the PANKRAS II Study. Eur J Epidemiol 22:577-588

Porta M, Ferrer-Armengou O, Pumarega J, López T, Crous-Bou M, Alguacil J et al (2008) Exocrine pancreatic cancer clinical factors were related to timing of blood extraction and influenced serum concentrations of lipids. J Clin Epidemiol 61:695-704

Porta M, Pumarega J, López T, Jariod M, Marco E, Grimalt JO (2009) Influence of tumor stage, symptoms and time of blood draw on serum concentrations of organochlorine compounds in exocrine pancreatic cancer. Cancer Causes Control 20:1893-1906

Porta M, Greenland S, Hernán M, dos Santos Silva I, Last M (eds) (2014) A dictionary of epidemiology, 6th edn. Oxford University Press, New York

Porta M, Pumarega J, Amaral AFS, Genkinger JM, Camargo J, Mucci L et al (2020) Influence of KRAS mutations, persistent organic pollutants, and trace elements on survival from pancreatic ductal adenocarcinoma. Environ Res 190:109781

Porta M, Gasull M, Pumarega J, Kiviranta H, Rantakokko P, RaaschouNielsen O et al (2021) Plasma concentrations of persistent organic pollutants and pancreatic cancer risk. Int J Epidemiol. https://doi. org/10.1093/ije/dyab115. Accessed 15 Oct 2021

Salcedo-Bellido I, Gutiérrez-González E, García-Esquinas E, Fernández de Larrea-Baz N, Navas-Acien A, Téllez-Plaza M et al (2021) Toxic metals in toenails as biomarkers of exposure: a review. Environ Res 197:111028

Signes-Pastor AJ, Gutiérrez-González E, García-Villarino M, Rodríguez-Cabrera FD, López-Moreno JJ, Varea-Jiménez E et al (2020) Toenails as a biomarker of exposure to arsenic: a review. Environ Res 2020:110286

Soler M, Malats N, Porta M, Fernandez E, Guarner L, Maguire A et al (1999) Medical conditions in patients with pancreatic and biliary diseases: validity and agreement between data from questionnaires and medical records. Dig Dis Sci 44:2469-2477

Sukumar A (2006) Human nails as a biomarker of element exposure. In: Ware GW, Nigg HN, Doerge DR (eds) Reviews of environmental contamination and toxicology, vol 185. Springer, New York, pp 141-177. https://link.springer.com/chapter/10.1007/0387-30638-2_5. Accessed 15 Oct 2021

Publisher's Note Springer Nature remains neutral with regard to jurisdictional claims in published maps and institutional affiliations.

\section{Authors and Affiliations}

\section{José Pumarega ${ }^{1,2,3} \cdot$ Judit Camargo $^{1,3} \cdot$ Magda Gasull, 2,3,4 $^{1, \text { Andrew F. Olshan }^{5} \cdot \text { Amr Soliman }^{6} \cdot \text { Yu Chen }}{ }^{7}$.

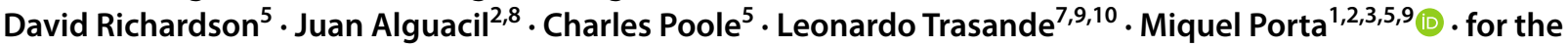 PANKRAS II Study Group}

1 Hospital del Mar Medical Research Institute (IMIM), Barcelona, Spain

2 CIBER de Epidemiología y Salud Pública (CIBERESP), Madrid, Spain

3 School of Medicine, Universitat Autònoma de Barcelona, Catalonia, Spain

4 Universitat Pompeu Fabra, Barcelona, Spain
5 Department of Epidemiology, Gillings School of Global Public Health, University of North Carolina, Chapel Hill, USA

6 Medical School of the City University of New York, New York, USA 
7 Departments of Environmental Medicine, and Population Health, New York University School of Medicine, New York, USA

8 Universidad de Huelva, Huelva, Spain
9 Department of Pediatrics, New York University School of Medicine, New York, USA

10 New York University College of Global Public Health, New York, USA 